\title{
Las potencialidades ilocutivas de las conjugaciones verbales en español: Una paráfrasis crítica y propositiva de "La enseñanza del verbo en la educación básica", de Hernán Urrutia
}

\author{
Illocutionary potential of verb conjugation in Spanish. \\ A critical and propositional paraphrase of "La enseñanza del verbo \\ en la educación básica" by Hernán Urrutia
}

\section{Claudia Rodríguez Monarca, Cecilia Quintrileo Llancao}

\author{
Instituto de Lingüística y Literatura \\ Facultad de Filosofía y Humanidades \\ Universidad Austral de Chile \\ cecilore@gmail.com \\ claudiar@uach.cl
}

Telf.: (56) 632221425

\begin{abstract}
RESUMEN
El presente artículo fija la atención en la relevancia de la lengua como instrumento de aprendizaje -particularmente las potencialidades ilocutivas de las conjugaciones verbales en español-, a partir de una revisión crítica del texto "La enseñanza del verbo en la educación básica" de Hernán Urrutia (1976). Este trabajo guarda vigencia tanto por su valor disciplinar, cuyos postulados están en sintonía con una perspectiva funcional de la enseñanza de la lengua, como por su carácter metodológico, al elaborar una propuesta didáctica para la enseñanza del verbo, centrada en la competencia comunicativa (lingüística, metalingüística, pragmática y discursiva). La segunda parte de este artículo propone reactivar aspectos metodológicos propuestos por Urrutia en una actividad proyectada para el aula y situada en el contexto escolar de educación básica, desde la integración de sus ejes a partir de un texto literario, que considera como corpus una canción de cuna de Gabriela Mistral.
\end{abstract}

Palabras clave: competencia comunicativa, canción de cuna, actos de habla, escritura, lectura

\begin{abstract}
The present article focuses on the relevance of language as a tool for learning - particularly the illocutionary potential of verb conjugation in Spanish - from a critical review of the text "La enseñanza del verbo en la educación basica” by Hernán Urrutia (1976). The importance of this research is related to its disciplinary value, based upon the functional approach for language teaching, and to its methodological value by elaborating a proposal for the teaching process of verb focused on the communicative ability (linguistics, metalinguistics, pragmatics and discourse). The second part of this paper encourages a reactivation of methodological aspects presented by Urrutia in a classroom oriented activity and located in the elementary education scenery by integrating its axis in a literary text which considers as corpus a lullaby by Gabriela Mistral.

Key words: communicative ability, lullaby, speech acts, writing, reading
\end{abstract}




\section{INTRODUCCIÓN}

El presente trabajo corresponde a una revisión crítica, pero a la vez propositiva y proyectiva, del artículo "La enseñanza del verbo en la educación básica" de Hernán Urrutia, publicado en Estudios Pedagógicos el año 1976. Lo hemos pensado como un homenaje al autor, por su trayectoria y por su legado intelectual. De esta manera, desde una visión pragmática del lenguaje, "homenajear" es un acto ilocutivo, específicamente de carácter expresivoasentivo ${ }^{1}$, en tanto que nosotras como emisoras manifestamos el respeto por su labor y un reconocimiento, en el sentido de "re-conocer", de volver a descubrir y tomar conciencia de la relevancia de la lengua como instrumento de aprendizaje -particularmente las potencialidades de los verbos- y de los modos en que puede ser trabajada en el aula.

Tomado el artículo original como hipotexto, como fuente principal, reelaboraremos un segundo texto, es decir un hipertexto, que se inspira en el anterior y se materializa a través de distintas estrategias, como la adaptación, la reescritura, la transposición o la traducción, para establecer una relación crítica, de homenaje o de comentario con el hipotexto (Genette, 1989) y, a su vez, reactivar aspectos metodológicos propuestos por Urrutia en una actividad proyectada para el aula y situada en el contexto escolar de educación básica, desde la integración de sus ejes a partir de un texto literario.

Una revisión crítica del artículo que data del año 1976, debe comenzar haciendo justicia sobre la vigencia que aún tiene, a pesar que la tendencia es considerar la obsolescencia del conocimiento, más aún cuando han pasado cuatro décadas. Pero hay varias razones por las que aún se debe tomar en cuenta como un referente importante en el estado del arte; una es de carácter científico y disciplinar: el asumir a sus antecesores otorga a la teoría linguiística el privilegio de su propia historia, es decir, fundar una tradición. Si los estudiosos actuales marginan los conocimientos a los que los lingüistas anteriores han contribuido, reniegan de su propio nacimiento sin, todavía, haber ejecutado el parricidio que legitimaría su desdén (Mariaca, 2007). El texto teórico dialoga y se conecta inevitablemente con otros textos anteriores, en esta especie de funcionamiento interdiscursivo y textual. Una segunda razón por la que reconocemos la vigencia de este artículo es de carácter metodológico. Urrutia elabora una propuesta didáctica para la enseñanza de la conjugación verbal en español:

El artículo no es de teoría lingüística ni de didáctica sino una aplicación didáctica de mecanismos lingüísticos básicos para superar un problema que se arrastra hasta la universidad entre los estudiantes de nuestro sistema educacional: el desconocimiento de las potencialidades de la conjugación del español (Urrutia, 1976: 97).

Los criterios que postula están en sintonía con una perspectiva funcional de la enseñanza de la lengua, disciplina que entonces, en la década del 70, estaba en sus albores y en período de emergencia. Entre las sugerencias metodológicas destaca: el carácter funcional de la lengua, la gradualidad del aprendizaje y de lo práctico a lo teórico, el carácter metacognitivo y metalingüístico, y un conjunto de actividades lúdicas y de producción de textos.

A continuación revisaremos algunos de estos aspectos metodológicos, desde una perspectiva funcional de la didáctica de la lengua, en contrapunto con las bases curriculares propuestas por el MINEDUC.

Bach y Harnish (1979: 42-44) reconocen entre los tipos de actos de habla expresivos, los Positivos Asentivos: "el hablante expresa su aprobación con respecto a lo propuesto por el interlocutor: Asentir, aceptar, aprobar, aplaudir, homenajear, alabar, adular". 


\section{LA DIDÁCTICA DE LA CONJUGACIÓN VERBAL DESDE UN ENFOQUE FUNCIONAL}

Considerando la vigencia de la propuesta metodológica de Urrutia, en este apartado revisamos la perspectiva funcional de la enseñanza de la lengua, a la luz de los avances en disciplinas tales como la pragmática (Levinson, 1985; Austin, 1962; Searle1969), la lingüística textual (Werlich, 1982; Beaugrade y Dressler, 1977), el análisis del discurso (Brown y Yule, 1983; Schiffrin, 1998; Van Dijk, 1985) y la etnografía de la comunicación (Hymes, 1971; 1972). Desde otro ángulo, debemos tener presente que el enfoque funcional constituye la orientación teórica adoptada en las Bases Curriculares y programas de estudio del MINEDUC para la enseñanza básica (2012).

El enfoque funcional puede integrarse en el paradigma comunicativo, marco común de principios lingüísticos y psicopedagógicos que sustentan los enfoques didácticos de enseñanza de la lengua más aceptados actualmente (Cassany, 1999). Una visión funcional de la enseñanza de la lengua pone énfasis en el lenguaje en uso, privilegiando el texto como máxima unidad de comunicación (Beaugrande y Dressler, 1977). Esta perspectiva didáctica reemplaza la visión historicista de la enseñanza de la lengua, centrada más bien en el conocimiento de la gramática, y con énfasis en la oración, asumiendo que los usuarios de la lengua no se expresan con frases u oraciones aisladas (López et al., 2008).

Asimismo, el enfoque funcional persigue el desarrollo de la competencia comunicativa, entendida como el conocimiento y la habilidad de los hablantes nativos para usar los recursos de su lengua, de modo lingüísticamente correcto y socialmente apropiado (Hymes, 1972). El concepto de competencia comunicativa, acuñado por Hymes (1971; 1972), de la etnografía de la comunicación, supera el concepto chomskiano de competencia lingüística, referido a la capacidad del hablante u oyente ideal para reconocer y producir una infinita cantidad de enunciados a partir de un número finito de unidades y reglas en una comunidad lingüística (Chomsky, 1965). De este modo, la competencia comunicativa se reserva para la lengua en uso, pasando a concebirse como parte de la competencia cultural, considerando la relevancia de una serie de principios o estrategias socioculturales, como la adecuación al contexto (De Beaugrande y Dressler, 1977), la selección del registro (Halliday, 1973), la capacidad de cooperación (Grice, 1975), entre otras. En otros términos, la competencia comunicativa integra una suma de competencias, que incluye las competencias lingüística, textual, sociolingüística, pragmática y la psicolingüística (Álvarez, 1995; Pilleux, 2001).

Desde la didáctica de la lengua, la competencia de comunicación se concibe como una forma de actividad humana, como un instrumento social desarrollado por y para los alumnos, para conseguir propósitos diversos (Cassany, 1999). De esta forma, el objetivo de la enseñanza de la lengua en la escuela es proporcionar a los alumnos el conocimiento, la práctica y la experiencia necesarias para comunicarse eficazmente en su lengua materna, tanto en forma oral como en forma escrita.

Hernán Urrutia (1976) propone un enfoque funcional para la didáctica de la conjugación en español, aspecto relevante e innovador en la enseñanza del verbo, y que ostenta plena vigencia si se consideran las potencialidades discursivas del verbo en el uso de la lengua. En la propuesta metodológica de Urrutia, hemos observado la importancia concedida a la competencia comunicativa, y particularmente a la competencia lingüística, la competencia pragmática y la competencia textual y discursiva. 


\subsection{LA COMPETENCIA LINGÜÍSTICA}

El problema planteado por Hernán Urrutia es el desconocimiento de las potencialidades de la conjugación en español en la enseñanza básica, aunque, a su juicio, se trataría de una situación que, en algunos casos, se arrastraría hasta la enseñanza superior.

Para hacer frente a esta problemática, propone una aplicación didáctica de mecanismos lingüísticos básicos con una metodología de carácter gradual, en dos fases de aprendizaje. La primera, focalizada sobre el paradigma de las tres conjugaciones (-ar, -er, -ir), enfatizando el conocimiento práctico de las magnitudes verbales y acentuando su valor metalingüístico: significado de los tiempos, persona, número y voz del verbo. Como se observa, se centra en la competencia lingüística, es decir, en los aspectos gramaticales del verbo.

En la primera fase de aprendizaje de la conjugación, Urrutia señala:

A) La primera hasta el $4^{\circ}$ año básico. En esta etapa hay que realizar múltiples ejercicios de oposición y conmutación para aprehender el valor metalingüístico de las tres conjugaciones (-ar, -er, -ir), el significado de los tiempos, modos de las personas, del número y de la voz en el verbo. // Esta etapa debe ser de conocimiento práctico de las magnitudes verbales. El estudio teórico y sistemático vendrá a posteriori. // Es la fase más importante, pues el conocimiento funcional debe estar de acuerdo con la teoría y nomenclatura que aprenderá después (1976: 97).

La primera etapa de aprendizaje se centra principalmente en ejercicios prácticos, que permitirían comprender las magnitudes verbales (tiempo, modo, número, voz del verbo), además de las distintas funciones que adoptan las formas verbales conjugadas (estado, acción, evento, proceso) en oraciones contextualizadas, lo que supone encauzar igualmente la competencia comunicativa, pragmática, la cual se aborda en detalle más adelante.

Por otra parte, Urrutia plantea una segunda fase:

B) La segunda desde el $5^{\circ}$ año básico. En esta etapa hay que continuar con los ejercicios anteriores, pero incorporando en forma sistemática la nomenclatura de descripción y clasificación gramatical de todos los modos y tiempos verbales, en las tres conjugaciones, destacando las irregularidades más conocidas, los sincretismos y variantes. Es recomendable en esta fase seguir los siguientes procedimientos:

Tras la selección de una forma de un texto proceder a realizar las oposiciones analizando los cambios de significado y sentido con referencia a fichas de clasificación de los paradigmas verbales (1976: 97).

La segunda fase de aprendizaje es de carácter teórico, a diferencia de la primera que es más práctica, y se relaciona igualmente con la competencia lingüística, centrándose particularmente en lo que llamaríamos una sub competencia metalingüística, pues propone el estudio teórico del verbo, incorporando la nomenclatura de descripción y clasificación gramatical de todos los modos y tiempos verbales y destacando las irregularidades más conocidas. Es necesario destacar que, en esta fase, la competencia metalingüística entra en interacción con la textual y discursiva, debido a que se propone la incorporación del texto, en contraste con la primera fase, donde se sugiere la práctica de las conjugaciones a través de la oración, en coherencia con un aprendizaje gradual. 


\subsection{LA COMPETENCIA PRAGMÁTICA}

La gradualidad del aprendizaje de la conjugación verbal es mediada por dos unidades de comunicación: la oración en contexto y el texto. Para la primera fase, Urrutia propone diseñar actividades, con oraciones contextualizadas ${ }^{2}$. Entre ellas destacan, por ejemplo, desde un proceso gradual:

a) Elegir una oración o contexto donde aparezca una forma verbal conocida o usada por los alumnos.

b) Realizar ejercicios de conmutación con las diferentes formas verbales para ver los cambios de significado y sentido.

c) La conmutación y oposición apuntarán a establecer sistemáticamente la oposición de tiempo, modo, persona, número y voz.

d) En un proceso de culminación se harían las combinaciones integradas con varias marcas o unidades de significado: tiempo, persona, modo, etc.

e) El valor trascendente de los morfemas verbales se varía en combinaciones y oposiciones con otros verbos que aportarían lexemas distintos y diferentes (1976: 98).

La incorporación del contexto es determinante en la conjugación verbal y en el desarrollo de una competencia pragmática, ya que permite que los alumnos tomen conciencia sobre la adecuación de las formas verbales a la situación comunicativa, la cual englobaría parámetros de tiempo y espacio, los participantes con sus características sociales, y las intenciones o finalidades de comunicación (Escandell, 1983).

Desde el plano pragmático, el verbo conjugado encierra la acción dirigida a la obtención de propósitos concretos. Desde la teoría de los actos de habla, la acción verbal es una característica de los verbos realizativos (Austin, 1962; Searle, 1969), los cuales conllevan la intención o finalidad comunicativa: por ejemplo, expresar un sentimiento como felicitar o insultar a alguien (actos expresivos), aseverar o negar un hecho (actos asertivos), dirigir órdenes o preguntas (actos directivos), realizar promesas (actos compromisorios), o declarar la culpabilidad de alguien sobre una acción (actos declarativos). Sin duda, la lista de estos verbos puede ser infinita y su uso depende de una serie de condiciones pragmáticas como el contenido proposicional (aspecto locutivo), la adecuación al contexto, las personas y finalidades verbales, pertinentes a las circunstancias (aspecto ilocutivo) y el efecto de las acciones verbales sobre las personas y el contexto (aspecto perlocutivo). Tal como propone Austin, 'decir es hacer', de manera que podemos considerar que la trilogía locutivo, ilocutivo, perlocutivo, constituiría adicionalmente otra potencialidad de la conjugación en español, la cual debe ponerse en práctica en la producción y comprensión de enunciados o textos.

\subsection{LA COMPETENCIA TEXTUAL Y DISCURSIVA}

La gradualidad del aprendizaje de la conjugación se observa a través de la propuesta de una segunda fase, donde se sugiere diseñar actividades incorporando el texto. Urrutia propone

Desde el punto de vista metalingüístico, en la primera fase de aprendizaje, convendría precisar que una oración aislada del contexto correspondería a una unidad meramente gramatical; en cambio, la emisión de una oración en contexto por un enunciador específico en una circunstancia determinada corresponde a una unidad de la pragmática. Para este segundo caso, preferimos reservar el término 'enunciado' (Escandell, 1983). 
"tras la selección de una forma de un texto proceder a realizar las oposiciones analizando los cambios de significado y sentido con referencia a fichas de clasificación de paradigmas verbales" (Urrutia, 1976: 98).

Como ya hemos señalado, desde el enfoque funcional, el texto constituye la máxima unidad de comunicación, lo que supone una competencia textual centrada en el conocimiento sobre los modos textuales y géneros discursivos (Ciapuscio, 2005) y en las habilidades para su producción e interpretación (Álvarez, 1995). De esta manera, en esta segunda fase, la enseñanza de los verbos debería considerar tanto la producción como el análisis de formas verbales en diferentes tipologías textuales y géneros literarios. Para efectos de este artículo queremos relevar entre los géneros literarios, la lírica, particularmente la destinada a los niños. En ella encontramos composiciones como las nanas o canciones de cuna.

A continuación presentamos un breve ejercicio para trabajar las conjugaciones verbales en el aula, que permita poner en acción las propuestas de Urrutia, tanto a nivel disciplinar como metodológico.

\title{
3. “DUÉRMETE MI NIÑO”... LA CONMUTACIÓN COMO JUEGO DE LAS CONJUGACIONES VERBALES. UNA PROPUESTA DIDÁCTICA PARA TRABAJAR CON LAS CANCIONES DE CUNA
}

\author{
El mar sus millares de olas \\ mece, divino. \\ Oyendo a los mares amantes, \\ mezo a mi niño. \\ "Meciendo", Gabriela Mistral
}

Como mencionamos al comienzo de este artículo, dos son las razones por las cuales el texto de Urrutia está vigente: una de carácter disciplinar y otra metodológica. A continuación haremos el ejercicio de ver cómo ambas se conjugan en una propuesta didáctica que considere los distintos ejes del subsector de Lenguaje y comunicación (lectura, escritura y comunicación oral), pensada para segundo ciclo de Enseñanza Básica, asumiendo como conocimiento previo la primera fase propuesta por Urrutia. De esta manera nos situamos en la segunda fase, más compleja en su gradualidad y que trabaja con textos y con contextos, y no con frases aisladas.

Las potencialidades de las conjugaciones verbales pueden ser estudiadas con mayor beneficio desde los actos de habla. Por esta razón hemos pensado en actividades relacionadas con las canciones de cuna, que son actos perlocutivos, acciones que se hacen en el decir y que modifican el estado del interlocutor (ya que la finalidad es que el niño se duerma y que afiance el vínculo con el adulto). El verbo cobra aquí una relevancia fundamental. A su vez, la elección de un corpus de esta naturaleza supone también la activación de conocimientos de la primera infancia, recuerdos de textos orales cantados por la madre en contextos comunicativos y afectivos particulares, que comienzan a configurar e inaugurar un itinerario literario con las canciones de cuna, las rondas, los cuentos y relatos orales; estos "libros sin páginas" a los que se refiere Yolanda Reyes (2005), que se escriben en la piel y en la memoria del niño. Habiendo pasado la primera infancia y construido en 
esta etapa ya sus caminos lectores, deberán reflexionar en torno a los propios y diversos recorridos en su formación como lectores y retomar contacto con estos textos.

Un adulto que mece a su hijo, le canta para que se duerma, pero también para transmitirle sus emociones, su alegría o su tristeza. Estos primeros textos oídos desde la complicidad del apego y el arrullo van a ir configurando ese "camino o recorrido lector" (Devetach, 2009). Pablo Bensaya (2006) complementa los argumentos de por qué trabajar con canciones de cuna, al referir que este género debe ser fortalecido por todas las vías posibles, enriqueciendo con nuestras propias acciones más elaboradas y conscientes estas formas que exceden el mundo del canto en sí mismo.

Metodológicamente proponemos trabajar las distintas competencias con énfasis en los distintos ejes, a partir de la canción de cuna "Apegado a mî", del libro de poemas Ternura, de Gabriela Mistral, publicado en Madrid, España, el año 1924, por la editorial Saturnino Calleja.

\author{
APEGADO A MÍ \\ Velloncito de mi carne, \\ que en mi entraña yo tejí, \\ velloncito friolento, \\ ¡duérmete apegado a mí! \\ La perdiz duerme en el trébol \\ escuchándole latir: \\ no te turben mis alientos, \\ ¡duérmete apegado a mí! \\ Hierbecita temblorosa \\ asombrada de vivir, \\ no te sueltes de mi pecho: \\ ¡duérmete apegado a mí! \\ Yo que todo lo he perdido \\ ahora tiemblo de dormir. \\ No resbales de mi brazo: \\ ¡duérmete apegado a mí!
}

La canción de cuna tiene estructuras versales, rítmicas y melódicas bien definidas, es decir, se trata de canciones isométricas e isorrítmicas, estrofas (la mayoría de cuatro versos) donde predominan ritmos binarios, ternarios y de compás libre.

En esta canción-poema, el uso de versos octosílabos y cláusulas rítmicas dactílicas de la canción de cuna crea el efecto rítmico que posibilita el sueño, junto a aspectos de la competencia comunicativa y de elementos paraverbales, como el volumen (bajo en este caso, casi de susurro) y las pausas. Pero la canción de cuna además tiene la particularidad que es en sí misma un conjunto de actos de habla perlocutivos, en que al ir diciendo (cantando) va provocando el efecto en su interlocutor, en su receptor. El poema es apelativo, se dirige a un niño. Esta apelación aparece marcada por una serie de verbos en modo imperativo que connotan un llamado a la acción: duérmete, no te sueltes, no resbales. 


\subsection{TALLER DE CONJUGACIÓN VERBAL: INDICACIONES PARA EL DOCENTE}

En el enfoque comunicativo de las base curriculares (2012), los Objetivos de Aprendizaje apuntan al desarrollo de las competencias comunicativas, que involucran conocimientos, habilidades y actitudes. De este modo, la asignatura de Lenguaje y comunicación busca desarrollar competencias que se adquieren participando en situaciones reales de lectura, escritura e interacción oral. Asimismo, el enfoque didáctico que deriva de esta visión concibe la interacción oral, la lectura y la escritura como situaciones comunicativas en que el conocimiento de la lengua y la corrección idiomática se ponen al servicio de la comunicación (MINEDUC). Desde el enfoque comunicativo y didáctico, las actividades elaboradas por el docente deberían integrar el conocimiento de las potencialidades sobre la conjugación verbal (el saber conocer), las habilidades (el saber hacer) y las actitudes (el saber ser y convivir) (Jabif, 2007).

En la propuesta de Urrutia, las actividades deberían desarrollarse desde lo práctico a lo teórico y desde lo metacognitivo a lo disciplinar, considerando el Objetivo de Aprendizaje de $5^{\circ}$ básico en cuanto a la conjugación en español: "Conjugar correctamente los verbos regulares al utilizarlos en sus producciones escritas". Además, es recomendable especificar la pertinencia de cada actividad según un eje específico, vinculado a un (sub)tipo de competencia comunicativa (metalingüística, pragmática, textual).

Desde lo práctico a lo teórico, hemos pensado en una actividad de Taller de Conjugación Verbal, en vinculación con un género específico, como es la canción de cuna. Como el taller práctico se fundamenta en los aspectos teóricos, consideramos además la pertinencia de un ítem, orientado a reforzar conocimiento previo, a través de una matriz didáctica de apoyo para el profesor.

El taller que proponemos se articula con una matriz didáctica y sugerencias metodológicas, que presentamos a continuación.

\section{a) Matriz didáctica}

La matriz didáctica contiene una serie de Indicaciones para el docente, que puede considerar como referencia para situar el aprendizaje de la conjugación verbal de acuerdo a las potencialidades del verbo (¿qué se aprende?), las habilidades involucradas en la actividad (¿cómo se aprende?) y las actitudes que se desarrollan (¿para qué se aprende?). Además, en esta matriz, el docente puede consignar el o los ejes del curriculum en el que se centra la actividad y la(s) competencia(s) comunicativa(s) pertinente(s). 
Figura 1: Matriz didáctica para la conjugación del tiempo/modo, eje de escritura

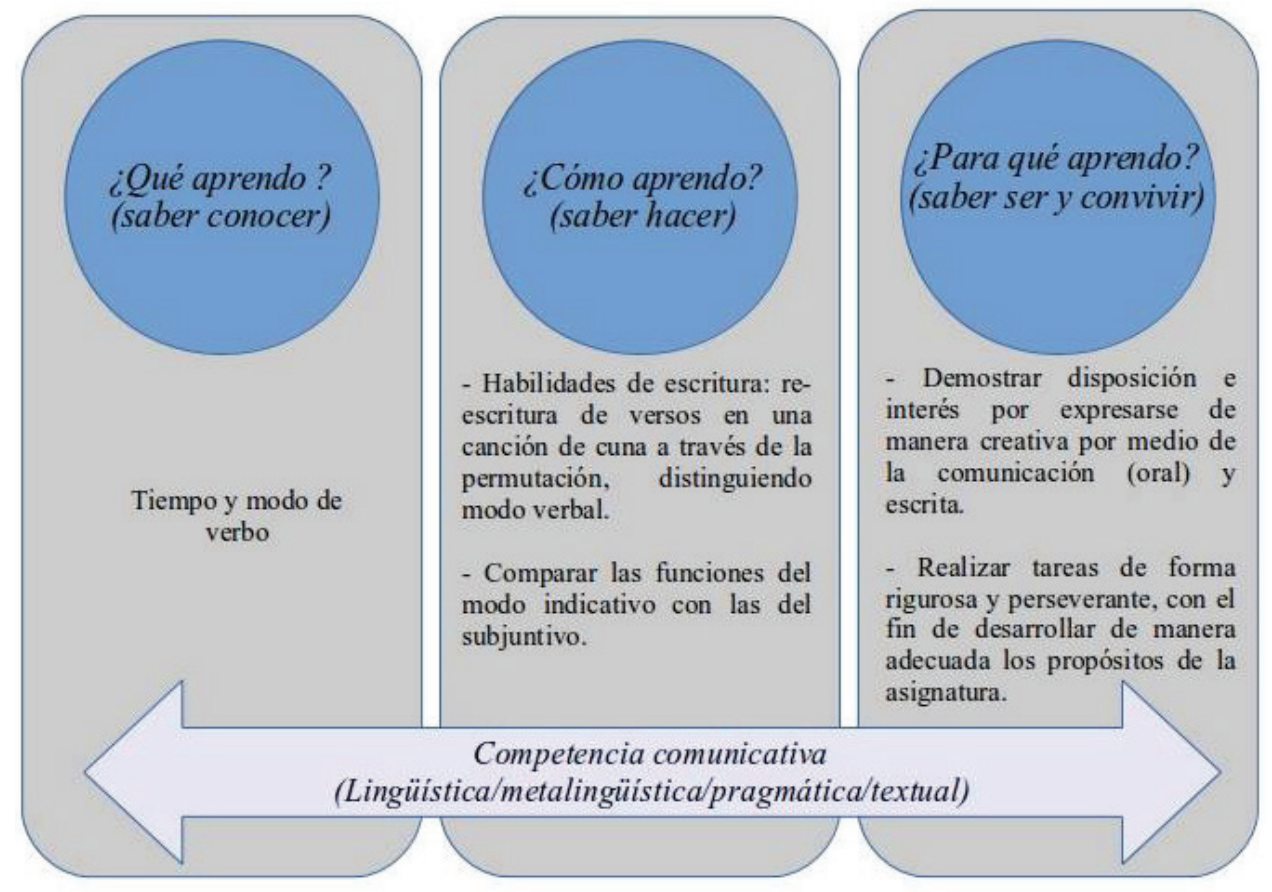

Como se observa en la figura, la matriz es un recurso de apoyo para el docente, donde visualiza la competencia comunicativa integrada con el conocimiento, las habilidades y las actitudes. Esta matriz puede complementarse con recursos adicionales considerados por el profesor, como, por ejemplo, una ficha funcional del verbo y guías de verbos del español, principalmente con los usos del indicativo y del subjuntivo en la subordinación.

Esta matriz puede considerarse también en los ejes de lectura y comunicación oral.

\section{b) Sugerencias metodológicas: taller de análisis y rescritura}

En un taller de análisis y reescritura en parejas, analizar y reconstruir el texto de Gabriela Mistral, permutando los tiempos verbales del Indicativo al modo Subjuntivo. Para ello, se puede presentar un repertorio léxico de verbos adicionales, que pueden incorporarse en la reconstrucción textual, activando conocimientos previos. El objetivo es comprender la potencialidad del modo verbal en un texto específico a través de la re-escritura, al distinguir la orientación de la acción o proceso sobre lo real o irreal. De esta manera, se pretende contribuir a los objetivos de aprendizaje (OA) en cuanto al dominio de las conjugaciones verbales (tiempo y modo), como al "Incorporar de manera pertinente en la escritura el vocabulario nuevo".

El taller puede utilizar como recurso una guía teórica de listado de verbos que exijan indicativo o subjuntivo. En el caso del subjuntivo, se sugieren verbos que significan 
entendimiento y percepción intelectual (creer, pensar, darse cuenta, enterarse, descubrir, dudar, esperar, exaltar) o verbos que expresan deseo, sentimiento, necesidad (querer, esperar, desear, encantar, ansiar, anhelar...). Estos deben anteponerse en los enunciados, para transformar el indicativo en subjuntivo (Marín y Ramírez, 2001). Ejemplo:

En la siguiente tabla se encuentran dos columnas. En la primera, se presenta el verso original de la canción de cuna de Gabriela Mistral; en la segunda, se debe reescribir el verso conmutando el modo.

\begin{tabular}{|l|l|}
\hline Verso original & Re-escritura del verso-conmutación del modo \\
\hline $\begin{array}{l}\text { velloncito friolento, } \\
\text { iduérmete apegado a mí! }\end{array}$ & $\begin{array}{l}\text { Por ejemplo: } \\
\text { •Velloncito friolento, [espero que] te duermas apegado a mí } \\
\bullet \text { Velloncito friolento [deseo que] te duermas apegado a mí }\end{array}$ \\
\hline $\begin{array}{l}\text { La perdiz duerme en el trébol } \\
\text { escuchándole latir }\end{array}$ & \\
\hline
\end{tabular}

Esta actividad puede realizarse igualmente con el número, según corresponda. Por ejemplo:

\begin{tabular}{|l|l|}
\hline Verso original & Re-escritura del verso-conmutación del género y número \\
\hline $\begin{array}{l}\text { Velloncito de mi carne, } \\
\text { que en mi entraña yo tejí, }\end{array}$ & $\begin{array}{l}\text { Velloncit(-a)(-s) de mi carne, } \\
\text { que en mi entraña yo tejí }\end{array}$ \\
\hline $\begin{array}{l}\text { La perdiz duerme en el trébol } \\
\text { escuchándole latir }\end{array}$ & \\
\hline
\end{tabular}

La conmutación del género y persona-número debe realizarse según sea el caso. En el primer verso puede aplicarse tanto al género como al número; en el segundo, el género femenino rige para ambos sexos, de manera que solo aplica la permutación del número, reforzando conocimiento previo sobre la adquisición de la lengua en cuanto a la concordancia artículo y sustantivo.

\section{c) Otras actividades}

En el eje escritura pueden considerarse otras actividades de escritura creativa, utilizando un repertorio variado de formas orientadas al logro de OA de $5^{\circ}$ año, como: "Escribir frecuentemente, para desarrollar la creatividad y expresar sus ideas, textos como poemas, diarios de vida, cuentos, anécdotas, cartas, blogs, etc". Por ejemplo, un taller de creación literaria, con canciones de cuna u otro género. Además del trabajo de conmutación de las conjugaciones verbales que plantea Urrutia también encontramos ejercicios de oposición. 
En este sentido se puede incorporar una actividad en que se contraste la acción de dormir de la canción de cuna con el tipo particular de las "mañanitas", que son canciones para despertar (por ejemplo, "Despierta, mi bien, despierta, / mira que ya amaneció. / Ya los pajaritos cantan, / la luna ya se ocultó”).

Este taller debe considerar la planificación del escrito y el proceso de edición, a través de la escritura guiada. Un taller de estas características, contribuye a dos OA específicos de $5^{\circ}$ básico: "Planificar sus textos: estableciendo propósito y destinatario, generando ideas a partir de sus conocimientos e investigación, organizando las ideas que compondrán su escrito", y "escribir, revisar y editar sus textos para satisfacer un propósito y transmitir sus ideas con claridad. Durante este proceso desarrollan las ideas, agregando información, emplean un vocabulario preciso y variado, y un registro adecuado, releen a medida que escriben, aseguran la coherencia y agregan conectores, editan, en forma independiente, aspectos de ortografía y presentación, utilizan las herramientas del procesador de textos para buscar sinónimos, corrigen ortografía y gramática, y dan formato". De este modo, se integraría el desarrollo de la competencia textual-discursiva a un eje específico, a través de la producción escrita, desde un género literario particular.

Hasta aquí hemos presentado un conjunto de actividades para trabajar las conjugaciones verbales a partir del eje de escritura, especialmente con el ejercicio de conmutación en una actividad de reescritura guiada. Pero además, se han sugerido otras actividades de escritura creativa que internalicen las distintas formas poéticas. Una propuesta que considere todos los ejes supone un aprendizaje integrado, por lo que quisiéramos complementar esta unidad con algunas actividades, al menos sugeridas, de los ejes de lectura y comunicación oral, que permitan articular la competencia pragmática y que pongan énfasis en aspectos actitudinales, tales como "demostrar disposición e interés por expresarse de manera creativa a través de las diversas formas de expresión oral y escrita". Recordemos que la competencia pragmática da cuenta de las dimensiones tanto lingüísticas como extralingüísticas, lo que supone las variables de contexto, participantes, intenciones de comunicación (actos de habla) y aspectos paraverbales que acompañan la palabra (volumen, tono, pausa, timbre y ritmo).

Para el eje de lectura, se sugiere primero leer en voz alta (puede ser individual, al unísono o en forma de canon) el texto propuesto u otras canciones de cuna (por ejemplo, la sección del mismo nombre del libro Ternura de Gabriela Mistral), incorporando los elementos paralingüísticos, de esta manera el estudiante adquiere conciencia fonológica, rítmica y melódica. De forma lúdica se podrían intercalar diferentes modalidades de lectura que pongan énfasis en uno de estos aspectos, por ejemplo las pausas (leer pausado, leer sin pausas), el volumen (leer en voz baja o en voz alta, gritando, susurrando). Con ello, damos cuenta del primer objetivo de aprendizaje propio para este nivel, contemplado en las Bases Curriculares, que persigue leer de manera fluida textos variados, apropiados a la edad del alumno, pronunciando las palabras con precisión y respetando la prosodia. Otro de los objetivos, el tercero de las bases, es leer y familiarizarse con un amplio repertorio de literatura para aumentar su conocimiento del mundo, desarrollar su imaginación y reconocer su valor social y cultural, para la toma de conciencia de los efectos de la lectura, en distintos contextos y situaciones de formalidad. Y en términos actitudinales, según consigna las Bases Curriculares, el objetivo que se persigue es "demostrar interés y una actitud activa frente a la lectura, orientada por el disfrute de la misma y por la valoración del conocimiento que se puede obtener a través de ella". 
Posterior a la lectura, se puede trabajar el eje de comunicación oral, centrándose en interacciones grupales para dialogar de manera afectiva y propositiva. Sugerimos una actividad donde los estudiantes puedan identificar la situación comunicativa, la relación afectiva entre los participantes y la finalidad de la canción como macro acto de habla, en tanto acto perlocutivo. Inmediatamente después, se les pide que compartan sus experiencias en torno a sus propios recorridos lectores, en una instancia de puesta en común frente al grupo curso. En el marco del enfoque comunicativo, el aprendizaje implica tanto el trabajo individual y reflexivo de cada alumno (con el objeto de reflexionar sobre sí mismo, sus ideas e intereses para desarrollar la autoconfianza y la autoestima) como la interacción y colaboración entre ellos. En este sentido, se pone énfasis también en los aspectos actitudinales (que derivan de los Objetivos de Aprendizaje Transversales), como "demostrar respeto por las diversas opiniones y puntos de vista y reconocer el diálogo como una herramienta de enriquecimiento personal y social".

\title{
4. CONCLUSIONES
}

En la revisión del artículo de Urrutia, hemos constatado el énfasis puesto sobre el carácter gradual, metalingüístico y metacognitivo de los aprendizajes en relación con la conjugación en español. En su propuesta metodológica, la gradualidad se observa en las dos fases de aprendizaje, que se potencian con una metodología anclada en lo metalingüístico y metacognitivo. Estos aspectos resultan fundamentales en el enfoque funcional en las bases curriculares del MINEDUC, donde el soporte disciplinar descansa en un metalenguaje, con miras a desarrollar conocimiento, habilidades y actitudes. Por lo tanto, la finalidad principal de la enseñanza de la lengua materna sería el desarrollo de las capacidades comprensivas, expresivas y metacomunicativas de los estudiantes, en el contexto de las conjugaciones verbales. En otros términos, la escuela debe enfatizar una didáctica orientada a proporcionar los recursos de expresión, comprensión y reflexión sobre los usos de la conjugación verbal, que permitan una utilización adecuada de la lengua en contextos variados, con diferente grado de formalización o planificación en sus producciones orales y escritas. Esto lo hemos querido mostrar a través de una propuesta didáctica pensada para segundo ciclo, con un corpus de canciones de cuna que, por su carácter perlocutivo, permite trabajar los verbos, ya que el sujeto poético, al ir diciendo (cantando) va provocando el efecto en su interlocutor.

\author{
El viento errabundo en la noche \\ mece los trigos. \\ Oyendo a los vientos amantes, \\ mezo a mi niño.
}

\section{REFERENCIAS BIBLIOGRÁFICAS}

Álvarez, G. (1995). Competencia discursiva y textual del hablante nativo. RLA, Revista de Lingüística Teórica y Aplicada, vol. 33: 5-14.

. (2001). Textos y discursos. Introducción a la lingüística del texto. Concepción: Universidad de Concepción.

Austin, J. (1962). How to do things with words. Londres: Oxford University Press. 
Estudios Pedagógicos, Número Especial 40 años: 137-149, 2016 LAS POTENCIALIDADES ILOCUTIVAS DE LAS CONJUGACIONES VERBALES EN ESPAÑOL: UNA PARÁFRASIS CRÍTICA Y PROPOSITIVA DE "LA ENSEÑANZA DEL VERBO EN LA EDUCACIÓN BÁSICA”, DE HERNÁN URRUTIA

Bach, K y Harnish, R (1979). Linguistic Communication and Speech Acts. Cambridge (Mass.): The MIT Press.

Beaugrade R. y W. Dressler (1977). Introducción a la lingüística del texto. Barcelona: Ariel.

Bensaya, P. (2006). La Canción de Cuna. Análisis y Dcoumentos Educativos. Presencias.Net, Abril 2006. Trcuperado desde: http://www.presencias.net/cantos/LaCancionDeCuna.html

Brown, G. y G. Yule (1983). Discourse Analysis. Cambridge: Cambridge University Press.

Ciapuscio, G. (2005). La noción de género en la Lingüística Sistémico Funcional y en la Lingüística Textual. Revista signos 38(57), 31-48. Recuperado el 21 de septiembre de 2016 desde https:// dx.doi.org/10.4067/S0718-09342005000100003

Cassany, D. (1999). Los enfoques comunicativos: elogio y crítica, Lingüística y Literatura, 36 (37), 11-33.

Chomsky, N. (1999[1965]). Aspectos de la teoría de la sintaxis. Barcelona: Gedisa.

Devetach, L. (2009). La construcción del camino lector. Córdoba: Comunicarte editorial.

Escandell, M.V. (1983). Introducción a la pragmática. Barcelona: Anthropos/UNED.

Fishman, J. (1970). Sociolinguistics: A brief introduction. Mass.: Newbury House.

Genette, G. (1989). Palimpsestos. La literatura en segundo grado. Madrid: Taurus.

Grice, H. P. (1975). Logic and conversation. En P. Cole y J. L. Morgan (Eds.), Speech acts (Syntax and Semantics, volume 3) (pp. 41-48). Nueva York: Academic Press.

Gumperz, J. y Hymes, D. (1964). The ethnography of communication. American Antropologist, vol. 66 (6), Parte 2: 137-154.

(1972). Directions in sociolinguistics. The ethnography of communication. Nueva York: Basil Blackwell.

Halliday, M.A.K. (1973). Explorations in Functions of Language. Londres: Edward Arnold.

Hymes, D. (1971). Competence and performance in linguistic theory. En R. Huxley y E. Ingram (Eds.) Acquisition of languages: Models and methods (pp. 3-28). New York: Academic Press.

. (1972). On communicative competence. En J.B. Pride y J. Holmes, Sociolinguistics (pp. 269-293). Londres: Penguin Books.

Jabif, L. (2007). La Docencia Universitaria Bajo un Enfoque de Competencias. Orientaciones prácticas para docentes. Valdivia: Universidad Austral de Chile.

Levinson, S. (1985). Pragmatics. Cambridge: Cambridge University Press.

López, M.I., I. Miranda y P. Mattioli (2008). Didáctica de la lengua. Unidad I. Recuperado el 30 de junio de 2016 desde http://www.feeye.uncu.edu.ar/web/lengua/did_lengua/anteriores/ Unidad_1_para_EGB.pdf

Mariaca, G. (2007). EL poder de la palabra. Ensayos sobre la modernidad de la crítica cultural hispanoamericana. Santiago: Tajamar Editores.

Marín, M. y Ramírez, P. (2001) Guía de gramática de la lengua española. Madrid: Espasa Calpe.

MINEDUC (2012). Bases curriculares Lenguaje y Comunicación. Recuperado el 04 de agosto de 2016 desde http://www.curriculumenlineamineduc.cl/605/w3-propertyvalue-49426.html.

Pilleux, M. (2001). Competencia comunicativa y análisis del discurso. Estudios filológicos, 36, 143 152. Recuperado desde https://dx.doi.org/10.4067/S0071-17132001003600010.

Reyes, Y. (2005). El libro que canta. Bogotá: Alfaguara.

. (2007). La casa imaginaria. Bogotá: Editorial Norma.

Schiffrin, D. (1998). Approaches to discourse. Mass.: Blackwell Publishers.

Searle, J. R. (1969). Speech acts: An essay in the philosophy of language. Cambridge: Cambridge University Press.

Urrutia, H. (1976) La enseñanza del verbo en la Educación Básica. Estudios Pedagógicos, vol. 1 (1), 97-98.

Van Dijk, T. (1985). Introduction: Discourse analysis as a new discipline. En Handbook in discourse analysis. Disciplines of discourse. Vol. 1. (pp. 1-10). Nueva York: Academic Press.

Werlich, E. (1982). A Text Grammar of English. Heidelberg: Quelle \& Meyer. 
